\title{
DISTRIBUSI INVERS GAMMA PADA INFERENSI BAYESIAN
}

\author{
Sugito $^{1}$, Dwi Ispriyanti ${ }^{2}$ \\ ${ }^{1,2}$ Staf Pengajar Program Studi Statistika FMIPA UNDIP
}

\begin{abstract}
One of the methods which can be used in statistical inferences is Bayesian inference. It is combine sample distribution and prior distribution, that can be resulted posterior distribution. In this article, sample distribution use univariate normal distribution. If prior distribution for variance with known mean is gamma inverse distribution, then posterior distribution is formed gamma inverse distribution. If Prior distribution use non-informative prior, then have the posterior distribution, by the marginal distribution of mean and varian. Also posterior distribution formed by gamma inverse distribution.
\end{abstract}

Keywords: Gamma Inverse Distribution, Posterior Distribution, Non-Informatif Prior

\section{Pendahuluan}

Statistik inferensia merupakan salah satu bidang statistik yang berhubungan dengan analisis data sampai pada peramalan atau penarikan kesimpulan mengenai suatu populasi. Pada umumnya statistik inferensia sepenuhnya menggunakan data yang diperoleh dari penelitian saat ini. Akan tetapi dalam kasus penelitian yang jarang terjadi misalnya penyakit kanker, aids atau flu burung, atau untuk kasus penelitian yang membutuhkan jangka waktu panjang seperti misalnya peluruhan radioaktif, inferensi statistik dari data sampel saja tidak cukup karena akan membutuhkan waktu yang lama untuk dapat menganalisis kasus tersebut. Jika inferensi dalam kasus seperti di atas menggunakan metode klasik (frequentis) yang didasarkan sepenuhnya pada informasi yang diperoleh melalui sampel, ini dapat menghasilkan error yang besar karena data sampel yang diambil terlalu sedikit, akibatnya inferensi yang dihasilkan nantinya kurang baik. Untuk mendapatkan inferensi yang lebih baik, akan lebih tepat jika data yang digunakan adalah data gabungan antara data sampel saat ini dengan data penelitian sebelumnya (data prior). Penggabungan data dilakukan dengan tujuan untuk meminimalkan tingkat kesalahan sehingga inferensi yang dihasilkan mendekati sempurna. Metode inferensi dengan menggunakan data sampel dan data prior disebut dengan metode Bayes.

Pada metode Bayes parameter yang digunakan merupakan variabel random yang mempunyai distribusi tertentu (distribusi prior). Distribusi prior adalah distribusi subyektif berdasarkan pada keyakinan seseorang dan dirumuskan sebelum data sampel diambil ${ }^{[7]}$. Distribusi sampel yang digabung dengan distribusi prior akan menghasilkan suatu distribusi yaitu distribusi posterior ${ }^{[5]}$. Distribusi posterior menyatakan derajat keyakinan seseorang mengenai suatu parameter setelah sampel diamati ${ }^{[7]}$. Jadi metode Bayes menggabungkan distribusi sampel dan distribusi prior sehingga dapat diperoleh distribusi posteriornya. Distribusi gamma mendapat namanya dari fungsi gamma yang sudah dikenal luas, dipelajari dalam banyak bidang matematika. Distribusi gamma yang ditransformasi akan menghasilkan distribusi inverse gamma ${ }^{[4]}$.

Dalam tulisan ini, akan dibahas mengenai:

a) Bagaimana menentukan distribusi posterior untuk inferensi variansi $\left(\sigma^{2}\right)$ dengan mean $(\theta)$ diketahui dengan distribusi prior invers gamma.

b) Bagaimana menentukan distribusi posterior marginal untuk non-informatif prior. 
Distribusi sampel yang digunakan dalam tulisan ini adalah distribusi normal univariat.

\section{Tinjauan Pustaka}

\subsection{Distribusi Normal Univariat}

Sebuah variabel random $X$ dikatakan mengikuti distribusi normal dengan mean $\theta$ dan variansi $\sigma^{2}$ dapat dilambangkan dengan $\mathrm{X} \sim \mathrm{N}\left(\theta, \sigma^{2}\right)$, jika memiliki fungsi kepadatan peluang dalam bentuk : $f(x)=\frac{1}{\sigma \sqrt{2 \pi}} \exp \left[-\frac{1}{2}\left(\frac{x-\theta}{\sigma}\right)^{2}\right]$

$$
\text { untuk }-\infty<x<\infty \text {, dengan }-\infty<\theta<\infty \text { dan } 0<\sigma<\infty^{[1]} \text {. }
$$

\subsection{Distribusi Gamma dan Invers Gamma}

Jika $X$ adalah variabel random berdistribusi gamma, dinotasikan dengan $X \sim \operatorname{GAM}(k, \theta)$ maka fungsi densitasnya dapat dinyatakan dalam bentuk:

$$
f(x)=\frac{1}{\theta^{k} \Gamma(k)} x^{k-1} \exp \left(-\frac{x}{\theta}\right) \quad x>0, k>0 \text { dan } \theta>0
$$

dan 0 untuk nilai $x$ yang lain.

Fungsi distribusi kumulatif untuk distribusi gamma dinyatakan dalam bentuk:

$$
F(x ; k ; \theta)=\int_{0}^{x} \frac{1}{\theta^{k} \Gamma(k)} x^{k-1} \exp \left(-\frac{x}{\theta}\right) d x
$$

Dengan melakukan transformasi $Y=g(X)=\frac{1}{X}$ maka: $y=\frac{1}{x} \Rightarrow x=\frac{1}{y}=g^{-1}(y)$

$$
\left|\frac{d}{d y} g^{-1}(y)\right|=\left|\frac{d}{d y}\left(\frac{1}{y}\right)\right|=\frac{1}{y^{2}}
$$

sehingga diperoleh:

$$
\begin{aligned}
f_{Y}(y) & =f_{X}\left[g^{-1}(y)\right]\left|\frac{d}{d y} g^{-1}(y)\right|=\frac{1}{\theta^{k} \Gamma(k)}\left(\frac{1}{y}\right)^{k-1} \exp \left(-\frac{1}{\theta y}\right) \frac{1}{y^{2}} \\
& =\frac{1}{\theta^{k} \Gamma(k)}\left(\frac{1}{y}\right)^{k+1} \exp \left(-\frac{1}{\theta y}\right)
\end{aligned}
$$

Jika $k=\alpha, \frac{1}{\theta}=\beta$ dan $x=y$, maka distribusi yang terbentuk adalah distribusi Invers Gamma seperti dinyatakan dalam persamaan:

$$
f(x)=\frac{\beta^{\alpha}}{\Gamma(\alpha)} x^{-(\alpha+1)} \exp \left(-\frac{\beta}{x}\right) \quad x>0, \alpha>0, \beta>0
$$

dan nol untuk nilai $x$ yang lain ${ }^{[8]}$.

\subsection{Distribusi Prior dan Distribusi Posterior}

\subsubsection{Distribusi Prior}

Distribusi prior adalah distribusi awal yang harus ditentukan terlebih dahulu sebelum merumuskan distribusi posteriornya. Distribusi prior adalah distribusi subyektif berdasarkan pada keyakinan seseorang dan dirumuskan sebelum data sampel diambil ${ }^{[7]}$. Distribusi prior ini dikelompokkan menjadi dua macam yaitu distribusi prior konjuget dan distribusi prior non-konjuget ${ }^{[2]}$. 
Bentuk distribusi prior konjuget merupakan pemberian bentuk distribusi prior yang sekawan atau sepola dengan bentuk distribusi dari hasil identifikasi pola datanya. Sedangkan distribusi prior non-konjuget adalah bentuk distribusi prior yang tidak sekawan dengan bentuk hasil identifikasi dari datanya ${ }^{[2]}$.

Misal didalam mengestimasi parameter $\theta$ tersedia informasi bahwa $\theta$ berubah sesuai dengan distribusi probabilitas tertentu. Jadi $\theta$ adalah variabel random yang menjalani harga-harga $\theta_{1}, \theta_{2}, \theta_{3}, \ldots \theta_{k}$ dengan distribusi probabilitas $p\left(\theta_{1}\right), \ldots, p\left(\theta_{k}\right)$. $P\left(\tilde{\theta}=\theta_{j}\right), \mathrm{j}=1,2, \ldots \mathrm{k}$ disebut distribusi prior untuk $\tilde{\theta}$, dimana $\tilde{\theta}=\left(\theta_{1}, \theta_{2}, \ldots, \theta_{k}\right)^{[6]}$.

\subsubsection{Distribusi Posterior}

Distribusi posterior adalah fungsi densitas bersyarat $\theta$ jika diketahui nilai observasi $x$. Ini dapat dituliskan sebagai: $f(\theta \mid x)=\frac{f(\theta, x)}{f(x)}$

Apabila $\theta$ kontinu, distribusi prior dan posterior $\theta$ dapat disajikan dengan fungsi kepadatan. Fungsi kepadatan bersyarat satu variabel random jika diketahui nilai variabel random kedua hanyalah fungsi kepadatan bersama dua variabel random itu dibagi dengan fungsi kepadatan marginal variabel random kedua. Tetapi fungsi kepadatan bersama $f(\theta, x)$ dan fungsi kepadatan marginal $f(x)$ pada umumnya tidak diketahui, hanya distribusi prior dan fungsi likelihood yang biasanya dinyatakan. Fungsi kepadatan bersama dan marginal yang diperlukan dapat ditulis dalam bentuk distribusi prior dan fungsi likelihood,

$$
f(\theta, x)=f(x \mid \theta) \cdot f(\theta)
$$

dimana $f(x \mid \theta)$ merupakan fungsi likelihood dan $f(\theta)$ merupakan distribusi prior ${ }^{[6]}$. Selanjutnya diketahui bahwa

$$
f(x)=\int_{-\infty}^{\infty} f(\theta, x) d \theta=\int_{-\infty}^{\infty} f(\theta) f(x \mid \theta) d \theta
$$

Sehingga dari fungsi kepadatan posterior untuk variabel random kontinu dapat ditulis sebagai :

$$
f(\theta \mid x)=\frac{f(\theta) f(x \mid \theta)}{\int_{-\infty}^{\infty} f(\theta) f(x \mid \theta) d \theta}
$$

\section{Hasil dan Pembahasan}

\subsection{Fungsi Likelihood dari Distribusi Normal}

Jika $X_{1}, X_{2}, \ldots, X_{n}$ adalah sampel random berdistribusi normal dengan densitas $f\left(x_{i} ; \theta, \sigma^{2}\right)$, maka fungsi likelihoodnya didefinisikan dengan:

$$
\begin{aligned}
L\left(\theta, \sigma^{2}\right) & =\prod_{i=1}^{n} f\left(x_{i} ; \theta, \sigma^{2}\right) \\
& =\prod_{i=1}^{n}\left(2 \pi \sigma^{2}\right)^{-1 / 2} \exp \left[-\frac{1}{2}\left(\frac{x_{i}-\theta}{\sigma}\right)^{2}\right]
\end{aligned}
$$




$$
\begin{aligned}
& =\left(2 \pi \sigma^{2}\right)^{-1 / 2} \exp \left[-\frac{1}{2 \sigma^{2}}\left(x_{1}-\theta\right)^{2}\right] \ldots .\left(2 \pi \sigma^{2}\right)^{-1 / 2} \exp \left[-\frac{1}{2 \sigma^{2}}\left(x_{n}-\theta\right)^{2}\right] \\
& =\left(2 \pi \sigma^{2}\right)^{-n / 2} \exp \left[-\frac{1}{2 \sigma^{2}} \sum_{i=1}^{n}\left(x_{i}-\theta\right)^{2}\right]
\end{aligned}
$$

\subsection{Distribusi Invers Gamma sebagai Distribusi Prior}

Sebuah variabel random $\sigma^{2}$ berdistribusi Invers Gamma dengan parameter $\alpha=0$ dan $\beta=0$ dinotasikan dengan $\sigma^{2} \sim I G(0,0)$, maka fungsi densitasnya dapat ditulis dalam $\operatorname{bentuk}^{[3]}: f\left(\sigma^{2}\right)=\frac{1}{\sigma^{2}} \quad, \sigma^{2}>0$.

Untuk mendapatkan bentuk distribusi posterior berdasarkan rumus distribusi posterior $f(\theta \mid x)=\frac{f(x \mid \theta) f(\theta)}{\int_{-\infty}^{\infty} f(x \mid \theta) f(\theta) d \theta}$, maka akan dicari terlebih dahulu fungsi likelihood dari distribusi sampel normal yaitu:

$$
\begin{aligned}
f\left(x \mid \sigma^{2}\right) & =\prod_{u=1}^{n} f\left(x_{u} \mid \sigma^{2}\right) \\
& =\prod_{u=1}^{n}\left(2 \pi \sigma^{2}\right)^{-\frac{1}{2}} \exp \left[-\frac{1}{2}\left(\frac{x_{u}-\theta}{\sigma}\right)^{2}\right] \\
& =\left(2 \pi \sigma^{2}\right)^{-\frac{n}{2}} \exp \left[-\frac{1}{2} \sum_{u=1}^{n}\left(\frac{x_{u}-\theta}{\sigma}\right)^{2}\right] \\
& =\left(2 \pi \sigma^{2}\right)^{-\frac{n}{2}} \exp \left[-\frac{1}{2 \sigma^{2}} \sum_{u=1}^{n}\left(x_{u}-\theta\right)^{2}\right] \\
& =(2 \pi)^{-\frac{n}{2}} \sigma^{-n} \exp \left[-\frac{1}{2 \sigma^{2}} \sum_{u=1}^{n}\left(x_{u}-\theta\right)^{2}\right]
\end{aligned}
$$

Setelah diperoleh bentuk fungsi likelihood dari distribusi sampel normal, dapat dirumuskan persamaan hasil kali fungsi likelihood distribusi sampel dengan distribusi prior sebagai berikut:

$$
\begin{aligned}
f\left(x \mid \sigma^{2}\right) f\left(\sigma^{2}\right)= & \left\{(2 \pi)^{-\frac{n}{2}}\left(\sigma^{2}\right)^{-\frac{n}{2}} \exp \left[-\frac{\sum_{u=1}^{n}\left(x_{u}-\theta\right)^{2}}{2 \sigma^{2}}\right]\right\}\left\{\frac{1}{\sigma^{2}}\right\} \\
& =\left\{(2 \pi)^{-\frac{n}{2}}\left(\sigma^{2}\right)^{-\frac{n}{2}} \exp \left[-\frac{\sum_{u=1}^{n}\left(x_{u}-\theta\right)^{2}}{2 \sigma^{2}}\right]\right\}\left(\sigma^{2}\right)^{-1}
\end{aligned}
$$




$$
=\left\{(2 \pi)^{-\frac{n}{2}}\left(\sigma^{2}\right)^{-\left(1+\frac{n}{2}\right)} \exp \left[-\frac{\sum_{u=1}^{n}\left(x_{u}-\theta\right)^{2}}{2 \sigma^{2}}\right]\right\}
$$

sehingga integral hasil perkalian antara fungsi likelihood distribusi sampel dengan distribusi prior adalah :

$$
\int_{-\infty}^{\infty} f\left(x \mid \sigma^{2}\right) f\left(\sigma^{2}\right) d \sigma^{2}=\int_{-\infty}^{\infty}\left\{(2 \pi)^{-\frac{n}{2}}\left(\sigma^{2}\right)^{-\left(1+\frac{n}{2}\right)} \exp \left[-\frac{\sum_{u=1}^{n}\left(x_{u}-\theta\right)^{2}}{2 \sigma^{2}}\right] d \sigma^{2}\right.
$$

Karena nilai $\sigma^{2}$ berjalan dari nol sampai tak hingga, maka integral hasil kali fungsi likelihood distribusi sampel dengan distribusi prior juga berjalan dari nol sampai tak hingga.

Misal $\sigma^{2}=q$

$$
\begin{aligned}
& \int_{0}^{\infty} f(x \mid q) f(q) d q=\int_{-\infty}^{\infty}\left\{(2 \pi)^{-\frac{n}{2}} q^{-\left(1+\frac{n}{2}\right)} \exp \left[-\frac{\sum_{u=1}^{n}\left(x_{u}-\theta\right)^{2}}{2 q}\right]\right\} d q \\
& =(2 \pi)^{-\frac{n}{2}} q^{-\left(1+\frac{n}{2}\right)}\left[\frac{\sum_{u=1}^{n}\left(x_{u}-\theta\right)^{2}}{2 q}\right]^{1-\frac{n}{2}}\left[\frac{\sum_{u=1}^{n}\left(x_{u}-\theta\right)^{2}}{2 q^{2}}\right]^{-1}
\end{aligned}
$$

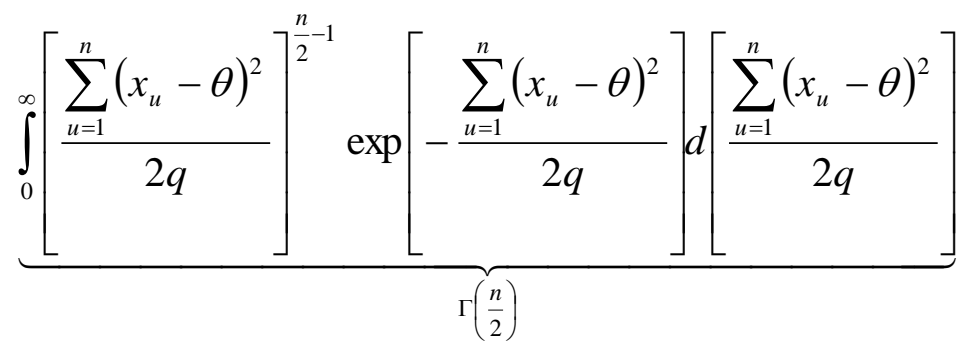

$$
\begin{aligned}
& =(2 \pi)^{-\frac{n}{2}}(q)^{-\left(1+\frac{n}{2}\right)} \Gamma\left(\frac{n}{2}\right) q\left[\frac{\sum_{u=1}^{n}\left(x_{u}-\theta\right)^{2}}{2 q}\right]^{-\frac{n}{2}}
\end{aligned}
$$

maka,

$$
\int_{0}^{\infty} f\left(x \mid \sigma^{2}\right) f\left(\sigma^{2}\right) d \sigma^{2}=(2 \pi)^{-\frac{n}{2}}\left(\sigma^{2}\right)^{-\left(1+\frac{n}{2}\right)} \Gamma\left(\frac{n}{2}\right) \sigma^{2}\left[\frac{\sum_{u=1}^{n}\left(x_{u}-\theta\right)^{2}}{2 \sigma^{2}}\right]^{-\frac{n}{2}}
$$




$$
\begin{aligned}
& f\left(\sigma^{2} \mid x\right)=\frac{(2 \pi)^{-\frac{n}{2}}\left(\sigma^{2}\right)^{-\left(1+\frac{n}{2}\right)} \exp \left[-\left[\frac{\sum_{u=1}^{n}\left(x_{u}-\theta\right)^{2}}{2 \sigma^{2}}\right]\right]}{(2 \pi)^{-\frac{n}{2}}\left(\sigma^{2}\right)^{-\left(1+\frac{n}{2}\right)} \Gamma\left(\frac{n}{2}\right) \sigma^{2}\left[\frac{\sum_{u=1}^{n}\left(x_{u}-\theta\right)^{2}}{2 \sigma^{2}}\right]^{-\frac{n}{2}}} \\
& =\left[\frac{\sum_{u=1}^{n}\left(x_{u}-\theta\right)^{2}}{2}\right]^{\frac{n}{2}}\left(\sigma^{2}\right)^{-\left(1+\frac{n}{2}\right)}\left[\Gamma\left(\frac{n}{2}\right)\right]^{-1} \exp \left[-\frac{\sum_{u=1}^{n}\left(x_{u}-\theta\right)^{2}}{2 \sigma^{2}}\right]
\end{aligned}
$$

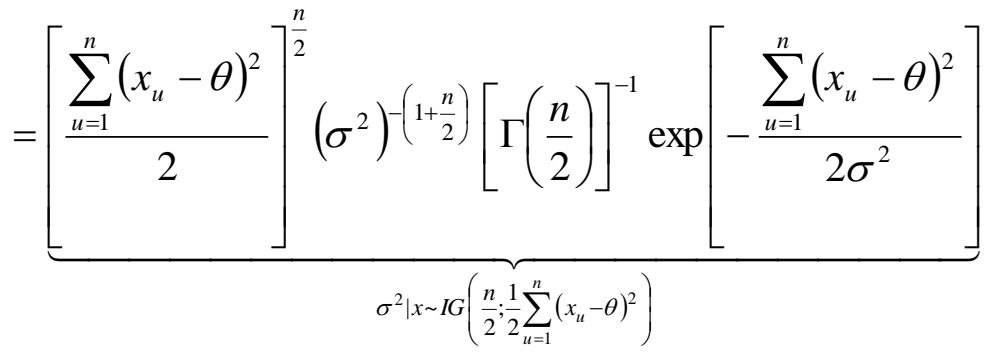

$$
\begin{aligned}
& f\left(\sigma^{2} \mid x\right) \sim I G\left[\frac{n}{2} ; \frac{1}{2} \sum_{u=1}^{n}\left(x_{u}-\theta\right)^{2}\right]
\end{aligned}
$$

Jadi distribusi posterior yang terbentuk adalah distribusi invers gamma.

\subsection{Non-Informatif Prior dari Distribusi Normal}

Distribusi non-informatif prior $f(\vartheta)$ dimana $\vartheta=\left(\theta, \sigma^{2}\right)$, diasumsikan bahwa $\theta$ dan $\sigma^{2}$ adalah independen sehingga

$$
f(\vartheta)=f(\theta) f\left(\sigma^{2}\right)^{[2]} .
$$

Mendapatakan distribusi non-informatif prior $f\left(\sigma^{2}\right)$

$$
\begin{aligned}
& f\left(X ; \theta, \sigma^{2}\right)=\frac{1}{\sigma \sqrt{2 \pi}} \exp \left[-\frac{1}{2}\left(\frac{X-\theta}{\sigma}\right)^{2}\right] \\
& \log f\left(X ; \theta, \sigma^{2}\right)=-\frac{1}{2} \log 2 \pi-\frac{1}{2} \log \sigma^{2}-\frac{(X-\theta)^{2}}{2 \sigma^{2}}
\end{aligned}
$$

Jika $u=\sigma^{2}$ maka

$$
\begin{aligned}
& \log f(X ; \theta, u)=-\frac{1}{2} \log 2 \pi-\frac{1}{2} \log u-\frac{(X-\theta)^{2}}{2 u} \\
& \frac{d \log f(X ; \theta, u)}{d u}=-\frac{1}{2 u}+\frac{(X-\theta)^{2}}{2 u^{2}} \\
& \frac{d^{2} \log f(X ; \theta, u)}{d u^{2}}=\frac{1}{2 u^{2}}-\frac{(X-\theta)^{2}}{u^{3}}=\frac{1}{2 \sigma^{4}}-\frac{(X-\theta)^{2}}{\sigma^{6}}
\end{aligned}
$$




$$
\begin{aligned}
& I\left(\sigma^{2}\right)=-E\left[\frac{d^{2} \log f(X ; \theta, u)}{d u^{2}}\right]=-\frac{1}{2 \sigma^{4}}+\frac{\sigma^{2}}{\sigma^{6}}=\frac{1}{2 \sigma^{4}} \\
& f\left(\sigma^{2}\right)=\sqrt{I\left(\sigma^{2}\right)}=\sqrt{\frac{1}{2 \sigma^{4}}} \propto \frac{1}{\sigma^{2}}
\end{aligned}
$$

Sedangkan nilai non-informatif prior untuk $f(\theta)=c$ (konstan) sehingga diperoleh

$$
f(\vartheta)=f(\theta) f\left(\sigma^{2}\right)=c \times \frac{1}{\sigma^{2}} \propto \frac{1}{\sigma^{2}}
$$

Jadi nilai non-informatif prior untuk $f(\vartheta)=\frac{1}{\sigma^{2}}$

\subsection{Distribusi Posterior}

Setelah mencari fungsi likelihood dan menentukan distribusi prior dari distribusi normal maka dapat dicari distribusi posteriornya

$$
f\left(\theta, \sigma^{2} \mid x\right)=\frac{L\left(\theta, \sigma^{2}\right) \times f(\vartheta)}{\int_{\sigma^{2}=0}^{\infty} \int_{\theta=-\infty}^{\infty} L\left(\theta, \sigma^{2}\right) \times f(\vartheta) d \theta d \sigma^{2}}
$$

Penjabaran untuk Pembilang:

$$
\begin{gathered}
L\left(\theta, \sigma^{2}\right) \times f(\vartheta)=\left(2 \pi \sigma^{2}\right)^{-n / 2} \exp \left[-\frac{1}{2 \sigma^{2}} \sum_{i=1}^{n}\left(x_{i}-\theta\right)^{2}\right] \times \frac{1}{\sigma^{2}} \\
=(2 \pi)^{-\frac{n}{2}}\left(\sigma^{2}\right)^{-\left(\frac{n}{2}+1\right)} \exp \left[-\frac{1}{2 \sigma^{2}} \sum_{i=1}^{n}\left(x_{i}-\theta\right)^{2}\right]
\end{gathered}
$$

Penjabaran untuk Penyebut:

$$
\int_{\sigma^{2}=0}^{\infty} \int_{\theta=-\infty}^{\infty} L\left(\theta, \sigma^{2}\right) \times f(\vartheta) d \theta d \sigma^{2}=\int_{\sigma^{2}=0}^{\infty} \int_{\theta=-\infty}^{\infty}\left(2 \pi \sigma^{2}\right)^{-n / 2} \exp \left[-\frac{1}{2 \sigma^{2}} \sum_{i=1}^{n}\left(x_{i}-\theta\right)^{2}\right] \times \frac{1}{\sigma^{2}} d \theta d \sigma^{2}
$$

Langkah 1

Integral terhadap $\theta$

$$
\begin{aligned}
& \int_{\theta=-\infty}^{\infty}\left(2 \pi \sigma^{2}\right)^{-n / 2} \exp \left[-\frac{1}{2 \sigma^{2}} \sum_{i=1}^{n}\left(x_{i}-\theta\right)^{2}\right] \times \frac{1}{\sigma^{2}} d \theta \\
& \text { dengan } \sum_{i=1}^{n}\left(x_{i}-\theta\right)^{2}=(n-1) s^{2}+n(\theta-\bar{x})^{2}=v s^{2}+n(\theta-\bar{x})^{2} \\
& \text { Jika } s^{2}=\frac{\sum_{i=1}^{n}\left(x_{i}-\bar{x}\right)^{2}}{n-1} \text { maka } \\
& =\int_{\theta=-\infty}^{\infty}(2 \pi)^{-n / 2}\left(\sigma^{2}\right)^{-\left(\frac{n}{2}+1\right)} \exp \left[-\frac{1}{2 \sigma^{2}}\left((n-1) s^{2}+n(\theta-\bar{x})^{2}\right)\right] d \theta \\
& =(2 \pi)^{-n / 2}\left(\sigma^{2}\right)^{-\left(\frac{n}{2}+1\right)} \int_{\theta=-\infty}^{\infty} \exp \left[-\frac{(n-1) s^{2}}{2 \sigma^{2}}\right] \exp \left[-\frac{n(\theta-\bar{x})^{2}}{2 \sigma^{2}}\right] d \theta
\end{aligned}
$$




$$
=(2 \pi)^{-n / 2}\left(\sigma^{2}\right)^{-\left(\frac{n}{2}+1\right)} \exp \left[-\frac{(n-1) s^{2}}{2 \sigma^{2}}\right] \int_{\theta=-\infty}^{\infty} \exp \left[-\frac{n(\theta-\bar{x})^{2}}{2 \sigma^{2}}\right] d \theta
$$

Dimana nilai $\int_{\theta=-\infty}^{\infty} \exp \left[-\frac{n(\theta-\bar{x})^{2}}{2 \sigma^{2}}\right] d \theta=\left[\frac{2 \pi \sigma^{2}}{n}\right]^{\frac{1}{2}}$

$$
\begin{aligned}
& =(2 \pi)^{-n / 2}\left(\sigma^{2}\right)^{-\left(\frac{n}{2}+1\right)} \exp \left[-\frac{(n-1) s^{2}}{2 \sigma^{2}}\right]\left[\frac{2 \pi \sigma^{2}}{n}\right]^{\frac{1}{2}} \\
& =(2 \pi)^{-n / 2}\left(\frac{2 \pi}{n}\right)^{\frac{1}{2}}\left(\sigma^{2}\right)^{-\left(\frac{n-1}{2}+1\right)} \exp \left[-\frac{(n-1) s^{2}}{2 \sigma^{2}}\right]
\end{aligned}
$$

Langkah 2

Integral terhadap $\sigma^{2}$

$$
\begin{aligned}
& \int_{\sigma^{2}=0}^{\infty}(2 \pi)^{-n / 2}\left(\frac{2 \pi}{n}\right)^{\frac{1}{2}}\left(\sigma^{2}\right)^{-\left(\frac{n-1}{2}+1\right)} \exp \left[-\frac{(n-1) s^{2}}{2 \sigma^{2}}\right] d \sigma^{2} \\
& =\int_{\sigma^{2}=0}^{\infty}(2 \pi)^{-n / 2}\left(\frac{2 \pi}{n}\right)^{\frac{1}{2}}\left(\sigma^{2}\right)^{-\left(\frac{(n-1)}{2}+1\right)} \exp \left[-\frac{(n-1) s^{2}}{2 \sigma^{2}}\right] d \sigma^{2} \\
& =(2 \pi)^{-n / 2}\left(\frac{2 \pi}{n}\right)^{\frac{1}{2}} \int_{\sigma^{2}=0}^{\infty}\left(\sigma^{2}\right)^{-\left(\frac{(n-1)}{2}+1\right)} \exp \left[-\frac{(n-1) s^{2}}{2 \sigma^{2}}\right] d \sigma^{2}
\end{aligned}
$$

Misal $A=\sigma^{2}$

$$
=(2 \pi)^{-n / 2}\left(\frac{2 \pi}{n}\right)^{\frac{1}{2}} \int_{A=0}^{\infty}(A)^{-\left(\frac{(n-1)}{2}+1\right)} \exp \left[-\frac{(n-1) s^{2}}{2 A}\right] d A
$$

Misal $B=\frac{(n-1) s^{2}}{2 A}$

$$
\begin{aligned}
& A=\frac{(n-1) s^{2}}{2 B} \frac{d A}{d B}=-\frac{(n-1) s^{2}}{2 B^{2}} d A=-\frac{(n-1) s^{2}}{2 B^{2}} d B \\
& \operatorname{Lim}_{A \rightarrow 0} B=\infty \\
& B=\infty \rightarrow A=0 \\
& =(2 \pi)^{-n / 2}\left(\frac{2 \pi}{n}\right)^{\frac{1}{2}} \int_{B=\infty}^{0}\left[\frac{(n-1) s^{2}}{2 B}\right]^{-\left(\frac{(n-1)}{2}+1\right)} \exp [-B]\left[-\frac{(n-1) s^{2}}{2 B^{2}}\right] d B \\
& =(2 \pi)^{-n / 2}\left(\frac{2 \pi}{n}\right)^{\frac{1}{2}} \int_{B=\infty}^{0}\left[\frac{v s^{2}}{2 B}\right]^{-\left(\frac{(n-1)}{2}+1\right)}\left[\frac{(n-1) s^{2}}{2 B}\right]\left[-\frac{1}{B}\right] \exp [-B] d B \\
& =(2 \pi)^{-n / 2}\left(\frac{2 \pi}{n}\right)^{\frac{1}{2}} \int_{B=\infty}^{0}\left[\frac{(n-1) s^{2}}{2 B}\right]^{-\left(\frac{(n-1)}{2}\right)}\left[-\frac{1}{B}\right] \exp [-B] d B \\
& =(2 \pi)^{-n / 2}\left(\frac{2 \pi}{n}\right)^{\frac{1}{2}}\left[\frac{(n-1) s^{2}}{2}\right]^{-\left(\frac{(n-1)}{2}\right)} \int_{B=\infty}^{0}\left[\frac{1}{B}\right]^{-\left(\frac{(n-1)}{2}\right)}\left[-\frac{1}{B}\right] \exp [-B] d B
\end{aligned}
$$




$$
\begin{aligned}
&=(2 \pi)^{-n / 2}\left(\frac{2 \pi}{n}\right)^{\frac{1}{2}}\left[\frac{(n-1) s^{2}}{2}\right]^{-\left(\frac{(n-1)}{2}\right)} \int_{B=0}^{\infty}\left[\frac{1}{B}\right]^{-\left(\frac{(n-1)}{2}\right)}\left[\frac{1}{B}\right] \exp [-B] d B \\
&=(2 \pi)^{-n / 2}\left(\frac{2 \pi}{n}\right)^{\frac{1}{2}}\left[\frac{(n-1) s^{2}}{2}\right]^{-\left(\frac{(n-1)}{2}\right)} \int_{B=0}^{\infty}(B)^{\frac{(n-1)}{2}-1} \exp [-B] d B \\
&=(2 \pi)^{-n / 2}\left(\frac{2 \pi}{n}\right)^{\frac{1}{2}}\left[\frac{(n-1) s^{2}}{2}\right]^{-\left(\frac{(n-1)}{2}\right)} \Gamma\left(\frac{(n-1)}{2}\right) \\
& \int_{\sigma^{2}=0}^{\infty} \int_{\theta=-\infty}^{\infty}\left(2 \pi \sigma^{2}\right)^{-n / 2} \exp \left[-\frac{1}{2 \sigma^{2}} \sum_{i=1}^{n}\left(x_{i}-\theta\right)^{2}\right] \times \frac{1}{\sigma^{2}} d \theta d \sigma^{2}=(2 \pi)^{-n / 2}\left(\frac{2 \pi}{n}\right)^{\frac{1}{2}}\left[\frac{(n-1) s^{2}}{2}\right]^{-\left(\frac{(n-1)}{2}\right)} \Gamma\left(\frac{(n-1)}{2}\right)
\end{aligned}
$$

Distribusi Posterior $f\left(\theta, \sigma^{2} \mid x\right)$

$$
\begin{aligned}
f\left(\theta, \sigma^{2} \mid x\right) & =\frac{(2 \pi)^{-n / 2}\left(\sigma^{2}\right)^{-\left(\frac{n}{2}+1\right)} \exp \left[-\frac{1}{2 \sigma^{2}} \sum_{i=1}^{n}\left(x_{i}-\theta\right)^{2}\right]}{(2 \pi)^{-n / 2}\left(\frac{2 \pi}{n}\right)^{\frac{1}{2}}\left[\frac{(n-1) s^{2}}{2}\right]^{-\left(\frac{(n-1)}{2}\right)} \Gamma\left(\frac{(n-1)}{2}\right)} \\
& =\left(\frac{n}{2 \pi}\right)^{\frac{1}{2}}\left[\frac{(n-1) s^{2}}{2}\right]^{\left(\frac{(n-1)}{2}\right)}\left[\Gamma\left(\frac{(n-1)}{2}\right)\right]^{-1}\left(\sigma^{2}\right)^{-\left(\frac{n}{2}+1\right)} \exp \left[-\frac{1}{2 \sigma^{2}} \sum_{i=1}^{n}\left(x_{i}-\theta\right)^{2}\right]
\end{aligned}
$$

\subsection{Distribusi Posterior Marginal untuk $\sigma^{2}$}

Jika $f\left(\theta, \sigma^{2} \mid x\right)$ dan merupakan distribusi posterior yang sudah terbentuk maka distribusi posterior marginal untuk $\sigma^{2}$ adalah:

$$
\begin{aligned}
f\left(\sigma^{2} \mid x\right) & =\int_{-\infty}^{\infty} f\left(\theta, \sigma^{2} \mid x\right) d \theta \\
f\left(\sigma^{2} \mid x\right) & =\int_{-\infty}^{\infty}\left(\frac{n}{2 \pi}\right)^{\frac{1}{2}}\left[\frac{(n-1) s^{2}}{2}\right]^{\left(\frac{(n-1)}{2}\right)}\left[\Gamma\left(\frac{(n-1)}{2}\right)\right]^{-1}\left(\sigma^{2}\right)^{-\left(\frac{n}{2}+1\right)} \exp \left[-\frac{1}{2 \sigma^{2}} \sum_{i=1}^{n}\left(x_{i}-\theta\right)^{2}\right] d \theta \\
& =\left(\frac{n}{2 \pi}\right)^{\frac{1}{2}}\left[\frac{(n-1) s^{2}}{2}\right]^{\left(\frac{(n-1)}{2}\right)}\left[\Gamma\left(\frac{(n-1)}{2}\right)\right]^{-1}\left(\sigma^{2}\right)^{-\left(\frac{n}{2}+1\right)} \int_{-\infty}^{\infty} \exp \left[-\frac{1}{2 \sigma^{2}} \sum_{i=1}^{n}\left(x_{i}-\theta\right)^{2}\right] d \theta \\
& =\left(\frac{n}{2 \pi}\right)^{\frac{1}{2}}\left[\frac{(n-1) s^{2}}{2}\right]^{\left(\frac{(n-1)}{2}\right)}\left[\Gamma\left(\frac{(n-1)}{2}\right)\right]^{-1}\left(\sigma^{2}\right)^{-\left(\frac{n}{2}+1\right)} \int_{-\infty}^{\infty} \exp \left[-\frac{1}{2 \sigma^{2}}\left((n-1) s^{2}+n(\theta-\bar{x})^{2}\right)\right] d \theta \\
& =\left(\frac{n}{2 \pi}\right)^{\frac{1}{2}}\left[\frac{(n-1) s^{2}}{2}\right]^{\left(\frac{(n-1)}{2}\right)}\left[\Gamma\left(\frac{(n-1)}{2}\right)\right]^{-1}\left(\sigma^{2}\right)^{-\left(\frac{n}{2}+1\right)} \exp \left[-\frac{(n-1) s^{2}}{2 \sigma^{2}}\right] \int_{-\infty}^{\infty} \exp \left[-\frac{n(\theta-\bar{x})^{2}}{2 \sigma^{2}}\right] d \theta
\end{aligned}
$$




$$
\begin{aligned}
& =\left(\frac{n}{2 \pi}\right)^{\frac{1}{2}}\left[\frac{(n-1) s^{2}}{2}\right]^{\left(\frac{(n-1)}{2}\right)}\left[\Gamma\left(\frac{(n-1)}{2}\right)\right]^{-1}\left(\sigma^{2}\right)^{-\left(\frac{n}{2}+1\right)} \exp \left[-\frac{(n-1) s^{2}}{2 \sigma^{2}}\right]\left[\frac{2 \pi \sigma^{2}}{n}\right]^{\frac{1}{2}} \\
& =\left(\frac{n}{2 \pi}\right)^{\frac{1}{2}}\left[\frac{(n-1) s^{2}}{2}\right]^{\left(\frac{(n-1)}{2}\right)}\left[\Gamma\left(\frac{(n-1)}{2}\right)\right]^{-1}\left(\sigma^{2}\right)^{-\left(\frac{n}{2}+1\right)} \exp \left[-\frac{(n-1) s^{2}}{2 \sigma^{2}}\right]\left[\frac{n}{2 \pi}\right]^{-\frac{1}{2}}\left(\sigma^{2}\right)^{\frac{1}{2}} \\
& =\left[\frac{(n-1) s^{2}}{2}\right]^{\left(\frac{(n-1)}{2}\right)}\left[\Gamma\left(\frac{(n-1)}{2}\right)\right]^{-1}\left(\sigma^{2}\right)^{-\left(\frac{(n-1)}{2}+1\right)} \exp \left[-\frac{(n-1) s^{2}}{2 \sigma^{2}}\right]
\end{aligned}
$$

Jadi nilai distribusi posterior marginal untuk $\sigma^{2}$ yang terbentuk $f\left(\sigma^{2} \mid x\right)$ adalah distribusi Invers Gamma dengan nilai $\alpha=\frac{(n-1)}{2}$ dan $\beta=\frac{(n-1) s^{2}}{2}$ $f\left(\sigma^{2} \mid x\right) \sim I G\left(\frac{(n-1)}{2}, \frac{(n-1) s^{2}}{2}\right)$ gamma dengan parameter $\alpha=\frac{n}{2}$ dan $\beta=\frac{1}{2} \sum_{u=1}^{n}\left(x_{u}-\theta\right)^{2}$.

\section{Kesimpulan}

1. Jika distribusi priornya adalah distribusi Invers Gamma $\sigma^{2} \sim I G(0,0)$, maka distribusi posterior yang diperoleh adalah distribusi Invers Gamma dengan parameter $\alpha=\frac{n}{2}$ dan $\beta=\frac{1}{2} \sum_{u=1}^{n}\left(x_{u}-\theta\right)^{2}$.

2. Nilai distribusi posterior marginal untuk varian $f\left(\sigma^{2} \mid x\right)$ menghasilkan nilai yang mendekati distribusi Invers Gamma dengan parameter $\frac{v}{2}$ dan $\frac{v s^{2}}{2}$.

\section{DAFTAR PUSTAKA}

1. Bain, L.J. and Engelhardt, M., Introduction to Probability and Mathematical Statistics, Second Edition, Duxbury Press, California, 1992.

2. Box, G.E.P and Tiao, G.C., Bayesian Inference In Statistical Analysis, Addision-Wesley Publishing Company, Inc, Philippines, 1973.

3. Dominici, F., Bayesian Methods - Third Term, 2002.

4. Dudewicz, E.J dan Mishra, S.N., Statistika Matematika Modern, ITB, Bandung, 1995.

5. Kuswandari, Inferensi Bayesian pada Model Linier Regresi, GDLHUB, Perpustakaan UNAIR: Surabaya, 2005, URL: http://adln.lib.unair.ac.id/go.

6. Soejoeti, Z dan Soebanar, Inferensi Bayesian, Karunika Universitas Terbuka, Jakarta, 1988.

7. Walpole, R. E dan Myers, R. H., Ilmu Peluang dan Statistika untuk Insinyur dan Ilmuwan, Terbitan Kedua, ITB, Bandung, 1986.

8 . . M.D. Anderson Cancer Center, Parameter Solver, Version 2.3 User's Guide, University of Texas, Department of Biostatistics and Applied Mathematics, URL: http://biostatistics.mdanderson.org/SoftwareDownload/. 